\title{
Upregulation of CKIP-1 inhibits high-glucose induced inflammation and oxidative stress in HRECs and attenuates diabetic retinopathy by modulating Nrf2/ARE signaling pathway: an in vitro study
}

Lan Zhang ${ }^{1,2}$, Jie $\mathrm{Yu}^{1,2}$, Mingxia Ye $\mathrm{e}^{1,2}$ and Hailan Zhao ${ }^{1,2^{*}}$

\begin{abstract}
Purpose: The aim of this study was to investigate the underlying mechanisms of diabetic retinopathy (DR) development.

Methods: Real-Time qPCR was used to detect Casein kinase 2 interacting protein-1 (CKIP-1) and Nuclear factor E2related factor 2 (Nrf2) mRNA levels. Western Blot was employed to detect protein levels. Malondialdehyde (MDA) assay kit, superoxide dismutase (SOD) kit and glutathione peroxidase (GSH-Px) kit were used to evaluate oxidative stress in high-glucose treated human retinal endothelial cells (HRECs). Calcein-AM/propidium iodide (PI) double stain kit was employed to detect cell apoptosis. Enzyme-linked ImmunoSorbent Assay (ELISA) was used to detect inflammation associated cytokines secretion. Co-immunoprecipitation (CO-IP) was performed to investigate the interactions between CKIP-1 and Nrf2. Luciferase reporter gene system was used to detect the transcriptional activity of Nrf2.

Results: CKIP-1 was significantly downregulated in either DR tissues or high-glucose treated HRECs comparing to the Control groups. Besides, high-glucose $(25 \mathrm{mM})$ inhibited HRECs viability and induced oxidative stress, inflammation associated cytokines (TNF-a, IL-6 and IL-1 $\beta$ ) secretion and cell apoptosis, which were all reversed by synergistically overexpressing CKIP-1 and aggravated by knocking down CKIP-1. Of note, we found that overexpressed CKIP-1 activated Nrf2/ARE signaling pathway and increased its downstream targets including HO-1, NQO-1, $\gamma G C S$ and SOD in high-glucose treated HRECs. Further results also showed that CKIP-1 regulated cell viability, oxidative stress, inflammation and apoptosis in high-glucose treated HRECs by activating Nrf2/ARE signaling pathway.
\end{abstract}

Conclusion: We concluded that overexpressed CKIP-1 alleviated DR progression by activating Nrf2/ARE signaling pathway.

Keywords: Diabetic retinopathy, Inflammation, Oxidative stress, CKIP-1, Nrf2/ARE signal pathway

\section{Introduction}

Diabetic retinopathy (DR) is a common complication of type 1 or type 2 diabetes mellitus (DM) [1], however, the underlying mechanisms are still not fully delineated.

\footnotetext{
*Correspondence: HailanZhaofrt@163.com

${ }^{1}$ Department of Ophthalmology, Zhejiang Provincial People's Hospital, No.158, Shangtang Road, Xiacheng District, Hangzhou 310014, Zhejiang, China

Full list of author information is available at the end of the article
}

Researchers found that 721 candidate genes might involve in DR progression [2]. In addition, researchers found that microRNA-183 [3] and miR-451a [4] also regulated DR progression. Besides, oxidative stress [5] and inflammation [6] were also pivotal for DR pathogenesis. For example, attenuation of oxidative stress by Gabapentin could help to alleviate DR in rats [7] and anti-inflammation drugs treatment attenuated DR in streptozotocin (STZ)-induced diabetic rats [8]. Hence 
targeting oxidative stress and inflammation might help to cure DR in clinic.

Casein kinase 2 interacting protein-1 (CKIP-1) participated in the regulation of multiple cell functions, such as cell proliferation $[9,10]$, apoptosis [11], inflammation $[12,13]$ and oxidative stress [11]. For example, CKIP-1 regulated the proliferative abilities of non-Hodgkin's lymphoma cells [10] and macrophages [9]. Besides, CKIP-1 also influenced cell apoptosis and oxidative stress, specifically, CKIP-1 alleviated oxygen-glucose deprivation/ reoxygenation induced and oxidative stress in hippocampal neurons [11]. In addition, CKIP-1 could regulate immune system, and CKIP-1 modulated inflammatory reactions by regulating $\mathrm{M} 1$ and $\mathrm{M} 2$ inflammatory macrophage polarization [13]. Since oxidative stress and inflammation are two important characteristics of DR, it is reasonable to speculate that CKIP-1 might be crucial for DR progression by regulating inflammation and oxidative stress. Notably, our preliminary experiments showed that CKIP-1 was aberrantly low-expressed in DR tissues and high-glucose treated HRECs comparing to the Control groups. The above results indicated that CKIP-1 might be the hub gene in DR progression by regulating oxidative stress and inflammation, and targeting CKIP-1 will provide new therapeutic agent for DR treatment.

Nuclear factor E2-related factor 2 (Nrf2)/antioxidant response element (ARE) pathway is the key regulator of oxidative stress [14], inflammation [15], cell proliferation [16] and apoptosis [17], and Nrf2/ARE signaling pathway was proved to be the downstream target of CKIP1 in cultured hippocampal neurons [11]. Interestingly, CKIP-1 ameliorated high-glucose induced expression of fibronectin and intercellular cell adhesion molecule-1 (ICAM-1) in glomerular mesangial cells by activating $N r f 2 / A R E$ signaling pathway [18]. In addition, Nrf2/ARE signaling pathway involved in the regulation of the cell damages caused by high-glucose treatment $[19,20]$. Therefore, the Nrf2/ARE signaling pathway might be the downstream target of CKIP-1 in DR development, however, the mechanisms are still unclear.

Taken together, we hypothesized that CKIP-1 might regulate DR progression by modulating Nrf2/ARE signaling pathway mediated cell proliferation, apoptosis, oxidative stress and inflammation. This study will uncover the underlying mechanisms of DR progression regulated by $C K I P-1$, and provide new therapeutic agents for DR treatment in clinic.

\section{Results}

The expression levels of CKIP-1 in DR tissues and high-glucose treated HRECs

CKIP-1 was reported to be closely related with highglucose induced diabetic nephropathy (DN) [18], hence we speculated that CKIP-1 might also participate in the development of DR. To validate the hypothesis, CKIP-1 expression levels were first evaluated in the clinical tissues. The results showed that CKIP-1 was downregulated in DR tissues comparing to the normal tissues (Fig. 1a, b). Of note, the results also showed that MDA levels was increased, SOD activity and GSH-PX activity were decreased in DR tissues comparing to the normal tissues (Fig. 1c), which suggested that oxidative stress played an important role in DR progression. In addition, the cellular results was in accordance with the clinical results, high-glucose treatment changed the morphology of HRECs from spindle-shape to round-shape (Fig. 1d), and decreased CKIP-1 levels in HRECs comparing to the control group (Fig. 1e, f). which indicated that cell viability and functions were affected by high-glucose treatment. Furthermore, the results showed that high-glucose $(25 \mathrm{mM})$ inhibited cell viability and promoted inflammation cytokines (TNF- $\alpha$, IL- 6 and IL-1 $\beta$ ) secretion in a time dependent manner (Additional file 1: Figure S1).

\section{Overexpressed CKIP-1 reverses the effects of high-glucose on HRECs viability, oxidative stress, inflammation and apoptosis}

We next explored the influences of overexpressed CKIP-1 on high-glucose treated HRECs in terms of cell viability, oxidative stress, inflammation and apoptosis. The results showed that high-glucose $(25 \mathrm{mM})$ significantly decreased CKIP-1 levels in HRECs comparing to the low-glucose group (5.5 mM), and pcDNA3.1-CKIP-1 was used to overexpress CKIP-1 in high-glucose $(25 \mathrm{mM})$ treated HRECs (Fig. 2a, b). The CCK-8 results showed that cell viability was inhibited by high-glucose $(25 \mathrm{mM})$ treatment, which was reversed by CKIP-1 overexpression (Fig. 2c). The further results showed that overexpressed CKIP-1 also abrogated the effects of high-glucose $(25 \mathrm{mM})$ on oxidative stress, inflammation and apoptosis (Fig. 2d-f). Specifically, overexpressed CKIP-1 abrogated the effects of high-glucose $(25 \mathrm{mM})$ on MDA levels, SOD activity and GSH-PX activity (Fig. 2d). High-glucose ( $25 \mathrm{mM})$ promoted secretions of inflammation associated cytokines (TNF- $\alpha$, IL-6 and IL-1 $\beta)$, which were abrogated by synergistically overexpressing CKIP-1 (Fig. 2d). The Calcein-AM/PI double stain assay results showed that overexpressed CKIP-1 abrogated the promoting effects of high-glucose $(25 \mathrm{mM})$ on cell apoptosis (Fig. 2e). The Western Blot results showed that high-glucose $(25 \mathrm{mM})$ promoted expressions of proapoptotic proteins (Bax and cleaved-Caspase 3 ) and inhibited anti-apoptotic protein $(\mathrm{Bcl}-2)$, which were reversed by CKIP-1 overexpression (Fig. 2f). 

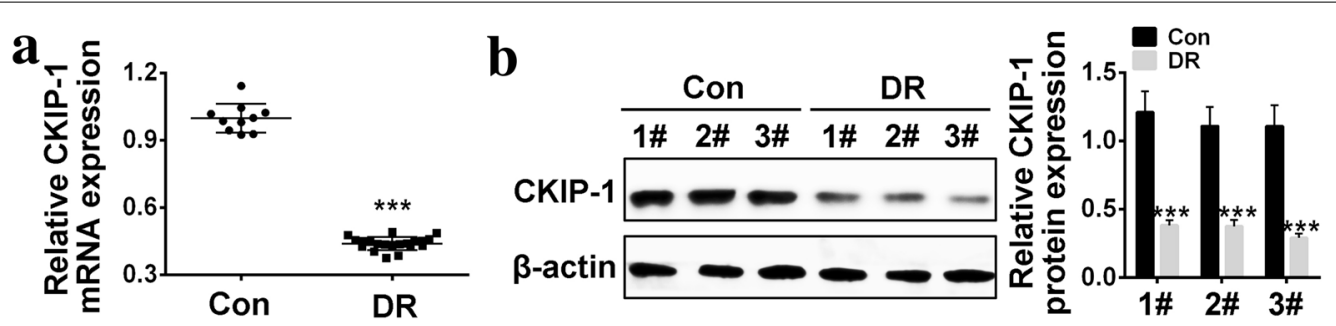

C
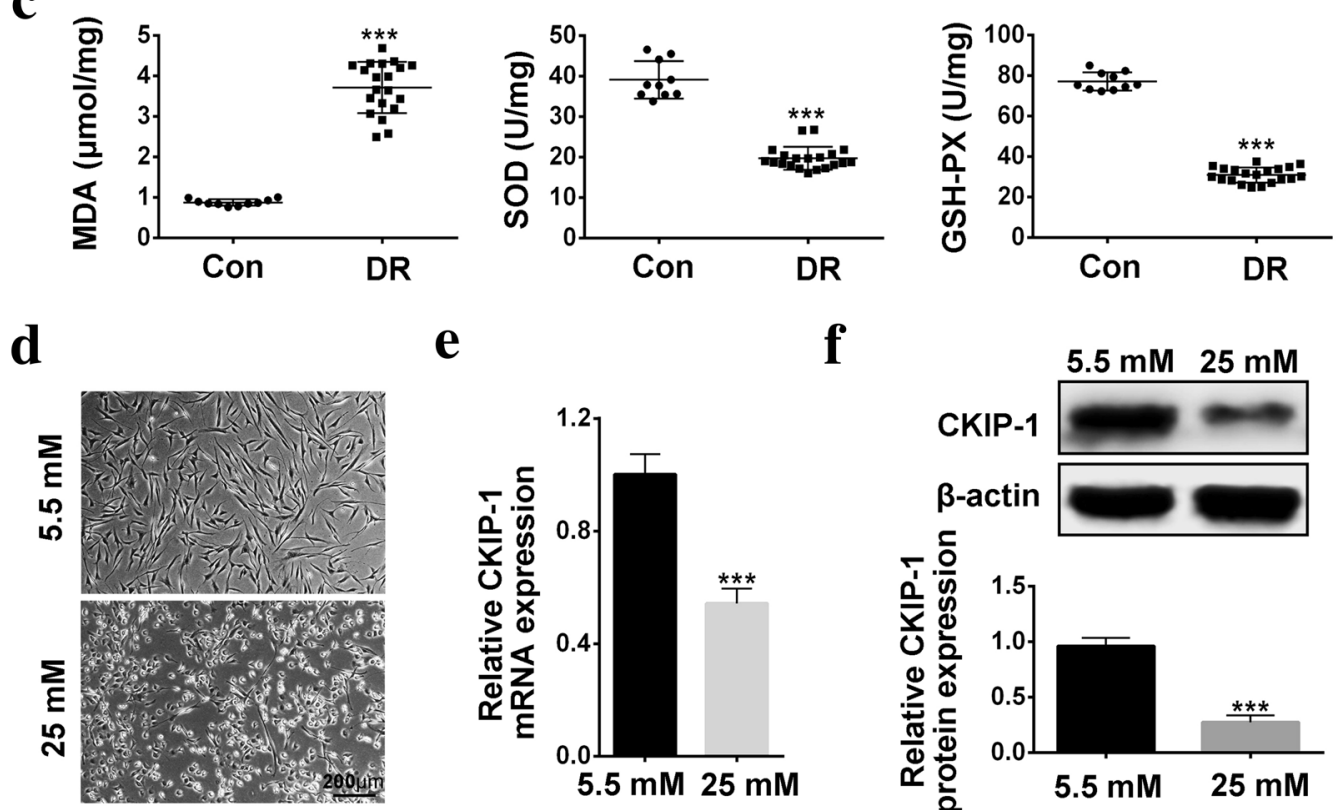

e

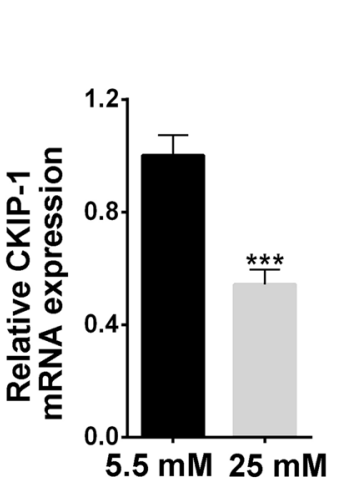

f

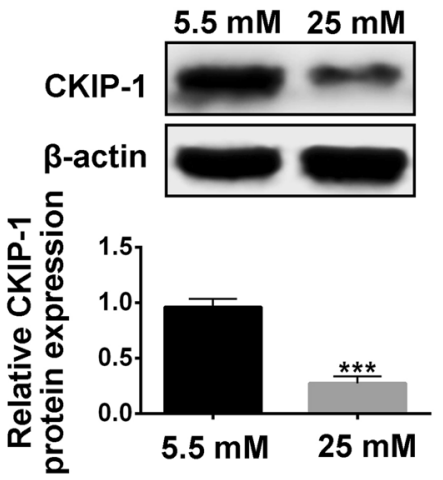

Fig. 1 CKIP-1 was downregulated in DR tissues and high-glucose treated HRECs. a Real-Time qPCR was used to detect CKIP-1 mRNA levels in DR tissues $(N=20)$ and normal tissues $(N=10)$. b Western Blot Was performed to detect CKIP-1 protein levels in DR tissues (The "1\#, 2\# and 3\#" represented 3 individual clinical specimens). c MDA assay kit was used to detect MDA levels, SOD kit was used to detect SOD activity and GSH-Px kit was used to detect GSH-Px activity in DR tissues respectively $(n=3)$. d The morphology of HRECs treated with low-glucose $(5.5 \mathrm{mM})$ and high-glucose ( $25 \mathrm{mM}$ ) (Scale bar is $200 \mu \mathrm{m})$. e Relative CKIP-1 mRNA levels in HRECs treated with low-glucose $(5.5 \mathrm{mM})$ and high-glucose (25 mM) was detected by Real-Time GPCR $(n=3)$. f Relative CKIP-1 protein levels in HRECs treated with low-glucose $(5.5 \mathrm{mM})$ and high-glucose $(25 \mathrm{mM})$ was detected by Western Blot (The "1\#, 2\# and 3\#" represented 3 individual repetitions). The data above in one experiments were repeated at least 3 times and performed as mean \pm standard deviation (SD), ${ }^{*} P<0.05,{ }^{* *} P<0.01$ and ${ }^{* * *} P<0.001$

Knock-down of CKIP-1 aggravated the effects of high-glucose on HRECs viability, oxidative stress, inflammation and apoptosis

We next explored the effects of CKIP-1 knock-down on high-glucose regulated HRECs viability, oxidative stress, inflammation and apoptosis. The results showed that we successfully established CKIP-1 knockdown HRECs (Fig. 3a, b). High-glucose treatment inhibited HRECs viability, which was aggravated by synergistically downregulating CKIP-1 (Fig. 3c). Similarly, knock-down of CKIP-1 enhanced the effects of high-glucose on SOD activity, GSH-PX activity and MDA levels (Fig. 3d), which indicated that synergistically knock-down of CKIP-1 aggravated the promoting effects of high-glucose treatment on HRECs oxidative stress. Aside from cell viability and oxidative stress, knock-down of CKIP-1 also enhanced the effects of high-glucose treatment on inflammation associated cytokines (TNF- $\alpha$, IL-6 and IL-1 $\beta$ ) secretion (Fig. 3d) and cell apoptosis (Fig. 3e, f).

The effects of overexpressed CKIP-1 on Nrf2/ARE signaling pathway activation in high-glucose treated HRECs

It was reported that $N r f 2 / A R E$ signaling pathway is the downstream target of CKIP-1 [21], hence we next explored whether $C K I P-1$ could regulate $N r f 2 / A R E$ signaling pathway in high-glucose treated HRECs. The CO-IP results showed that CKIP-1 interacted with $N r f 2$, which could further form CKIP-1-Nrf2 protein complex in HRECs (Fig. 4a, b). We also found that high-glucose treatment significantly inhibited the transcriptional activity of $N r f 2$, which was reversed by overexpressing 
$\mathbf{a}$

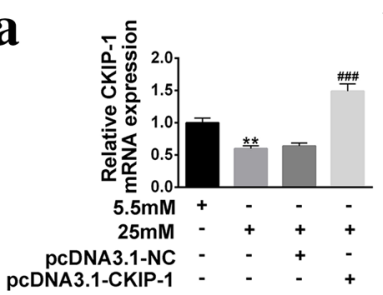

d
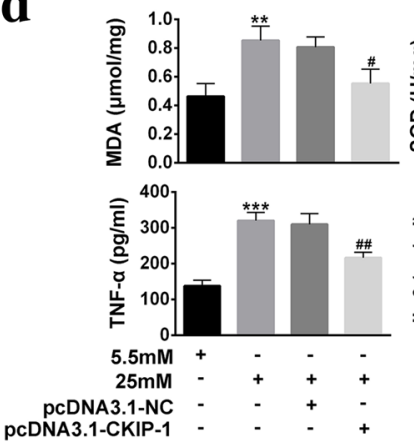

e
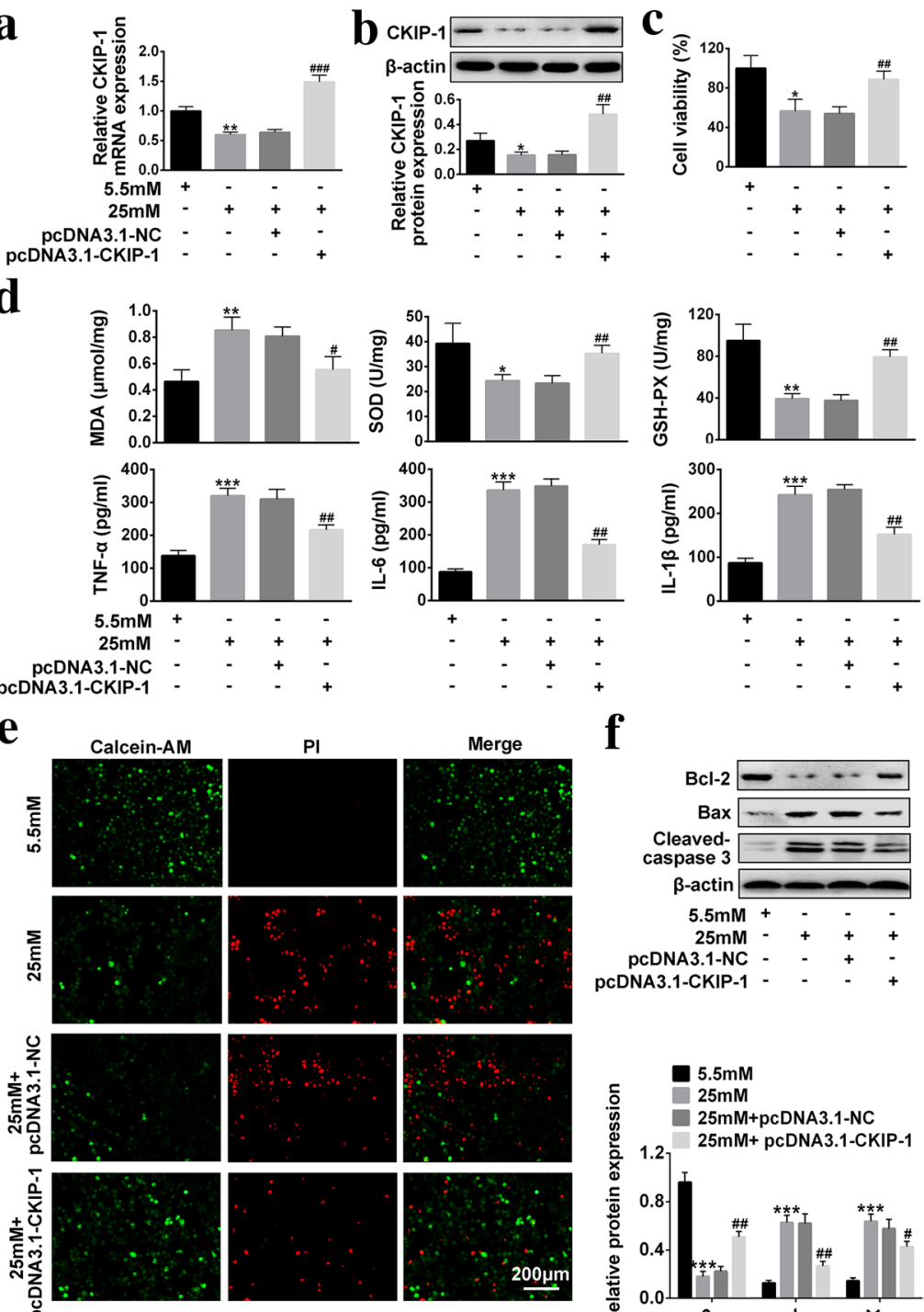

Merge

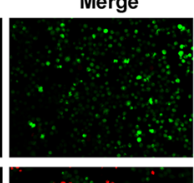

f
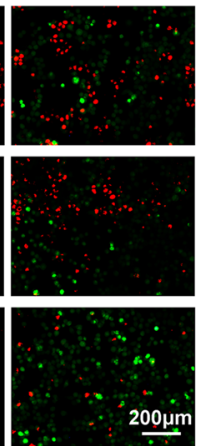

$200 \mu \mathrm{m}$

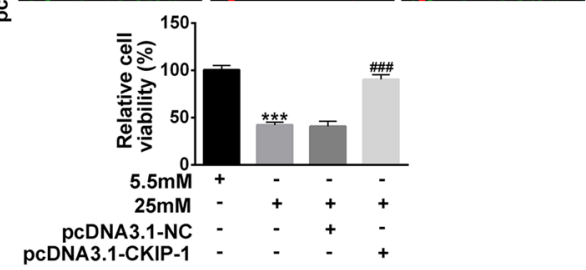

Fig. 2 The effects of overexpressed CKIP-1 on high-glucose treated HRECs in terms of cell viability, oxidative stress, inflammation and apoptosis. a Relative CKIP-1 mRNA levels were detected by Real-Time qPCR $(n=3)$. b Relative CKIP-1 protein levels were detected by Western Blot ( $\mathrm{n}=3)$. $\mathbf{c}$ CCK-8 assay kit was utilized to detect cell viability $(n=3)$. d MDA levels, SOD activity and GSH-PX activity were detected by MDA assay kit, SOD kit abd GSH-PX kit respectively $(n=3)$. Inflammation associated cytokines (TNF-a, IL-6 and IL-1 3 ) were detected by ELISA ( $n=3$ ). e Calcein-AM/PI double stain kit was employed to detect cell apoptosis (Scale bar is $200 \mu \mathrm{m}$ ). f Apoptosis associated proteins (BCl-2, Bax and Cleaved Caspase 3) were detected by Western Blot $(n=3)$. The data above in one experiments were repeated at least 3 times and performed as mean \pm standard deviation (SD), ${ }^{*} P<0.05,{ }^{* *} P<0.01$ and ${ }^{* *} P<0.001$

CKIP-1 (Fig. 4c). Of note, our results showed that highglucose treatment affected the cellular localization of Nrf2 in HRECs (Fig. 4d, e). Specifically, high-glucose treatment increased cytoplasm $\mathrm{Nrf} 2$ levels, but decreased nuclear Nrf2 levels, which were significantly reversed by CKIP-1 overexpression (Fig. $4 \mathrm{~d}, \mathrm{e}$ ). The results indicated that overexpressed CKIP-1 promoted Nrf2 translocation from cytoplasm to nuclear and facilitating the activation 

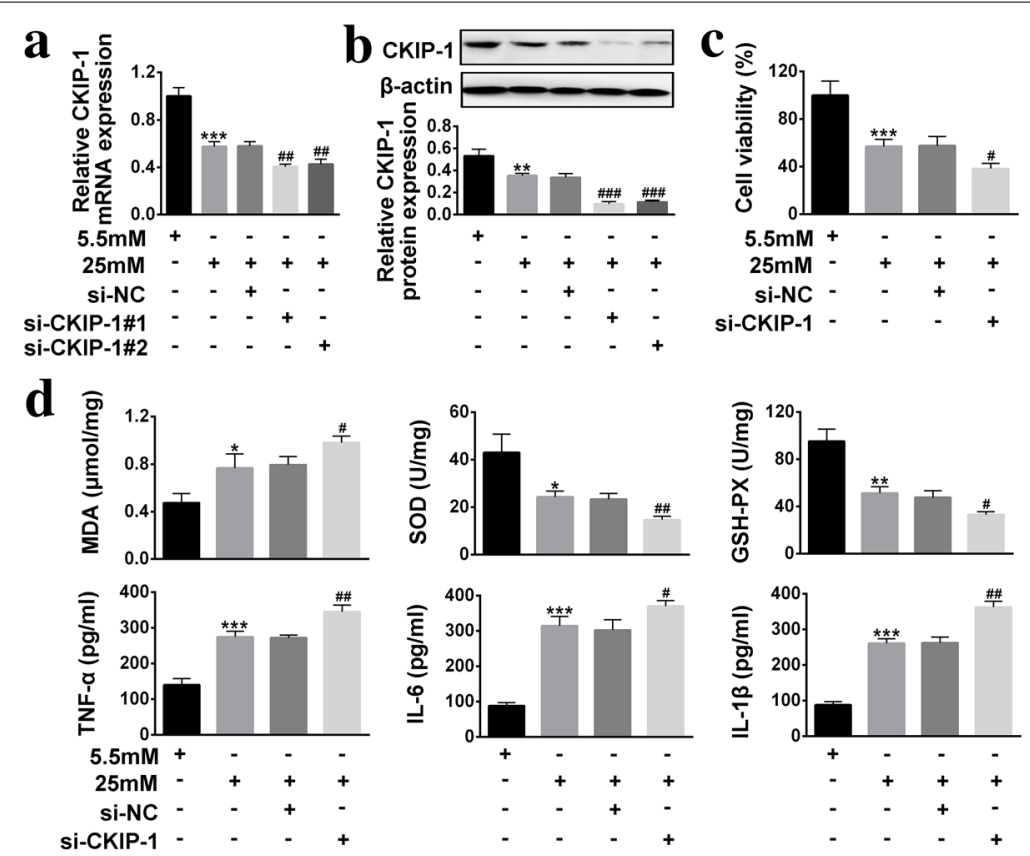

e
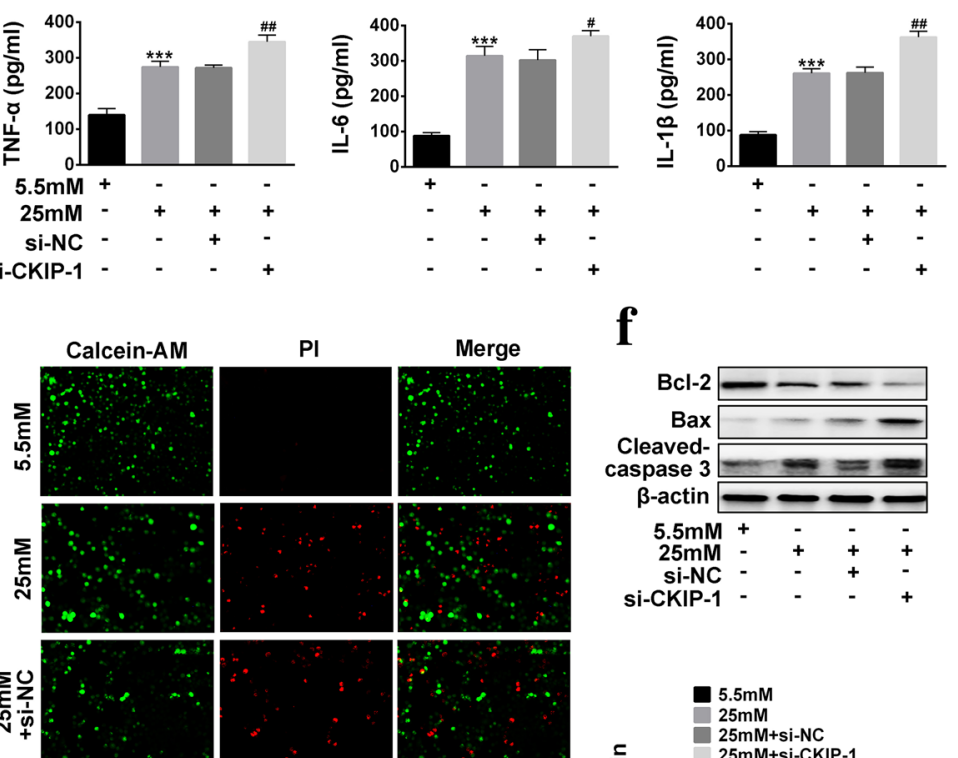

Merge

f
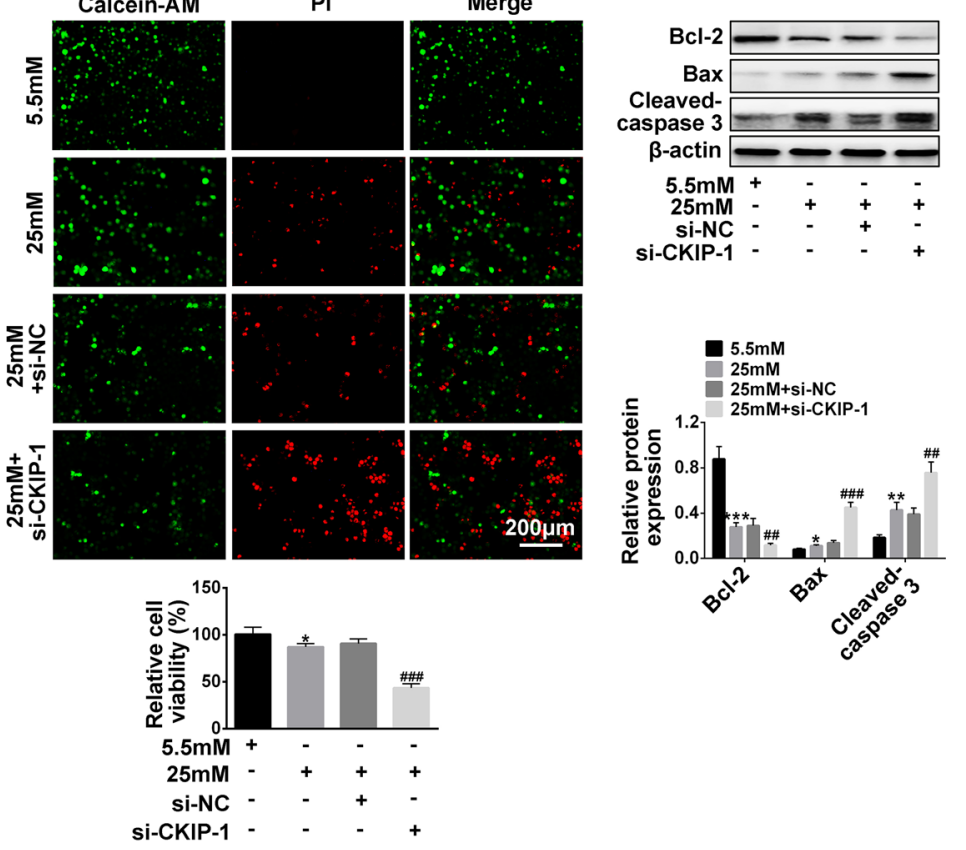

Fig. 3 The effects of CKIP-1 knock-down on high-glucose treated HRECs in terms of cell viability, oxidative stress, inflammation and apoptosis. a Relative CKIP-1 mRNA levels were detected by Real-Time qPCR $(n=3)$. b Relative CKIP-1 protein levels were detected by Western Blot $(n=3)$. c CCK-8 assay kit was utilized to detect cell viability. $\mathbf{d}$ MDA levels, SOD activity and GSH-PX activity were detected by MDA assay kit, SOD kit abd GSH-PX kit respectively $(n=3)$. Inflammation associated cytokines (TNF- $a$, IL-6 and IL-1 $\beta$ ) were detected by ELISA $(n=3)$. e Calcein-AM/PI double stain kit was employed to detect cell apoptosis (Scale bar is $200 \mu \mathrm{m}$ ). f Apoptosis associated proteins (BCl-2, Bax and Cleaved Caspase 3) were detected by Western Blot $(n=3)$. The data above in one experiments were repeated at least 3 times and performed as mean \pm standard deviation $(S D),{ }^{*} P<0.05$, ${ }^{* *} P<0.01$ and ${ }^{* *} P<0.001$ 
of downstream targets of Nrf2. Accordingly, our results proved that high-glucose inhibited the expressions of the downstream targets of Nrf2 (including HO-1, NQO-1, $\gamma G C S$ and $S O D$ ) in HRECs, which were also reversed by synergistically transfecting cells with CKIP-1 overexpression vectors (Fig. 4f).
CKIP-1 regulated cell viability, oxidative stress, inflammation and apoptosis of high-glucose treated HRECs by activating Nrf2/ARE signaling pathway

Nrf2/ARE signaling pathway has been reported to participate in the regulation of cell viability [21], oxidative stress [22], inflammation [23] and apoptosis [22], which has also been proved to be regulated by CKIP- 1 in the

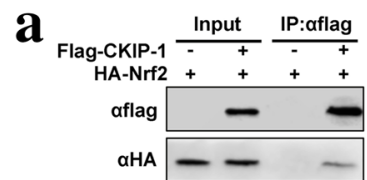

C
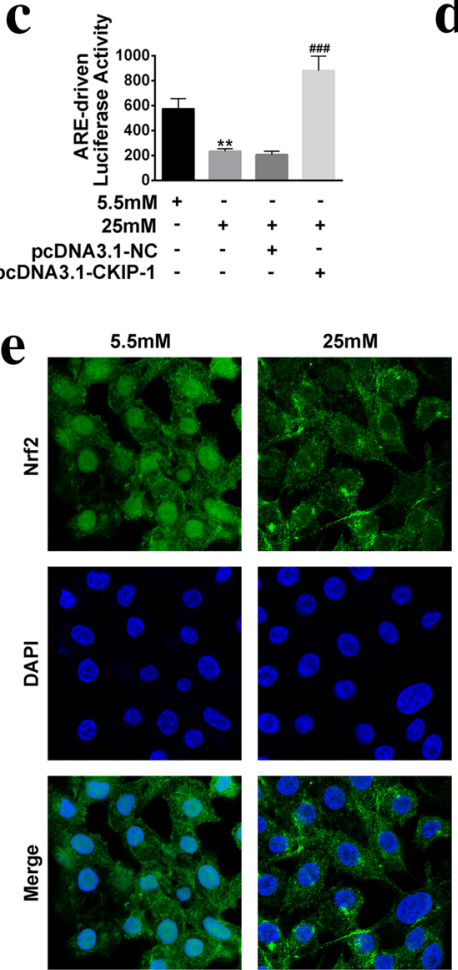

$\mathbf{f}$

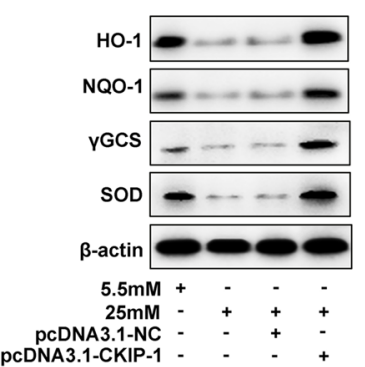

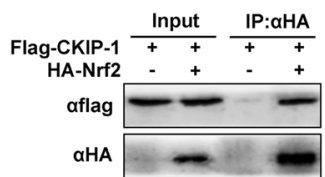

d

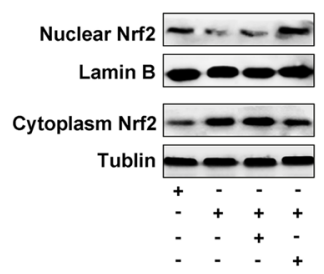

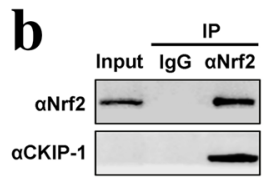

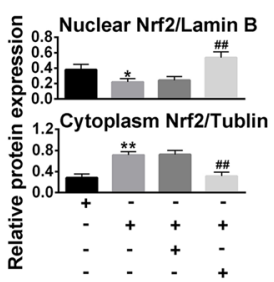

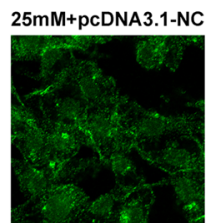
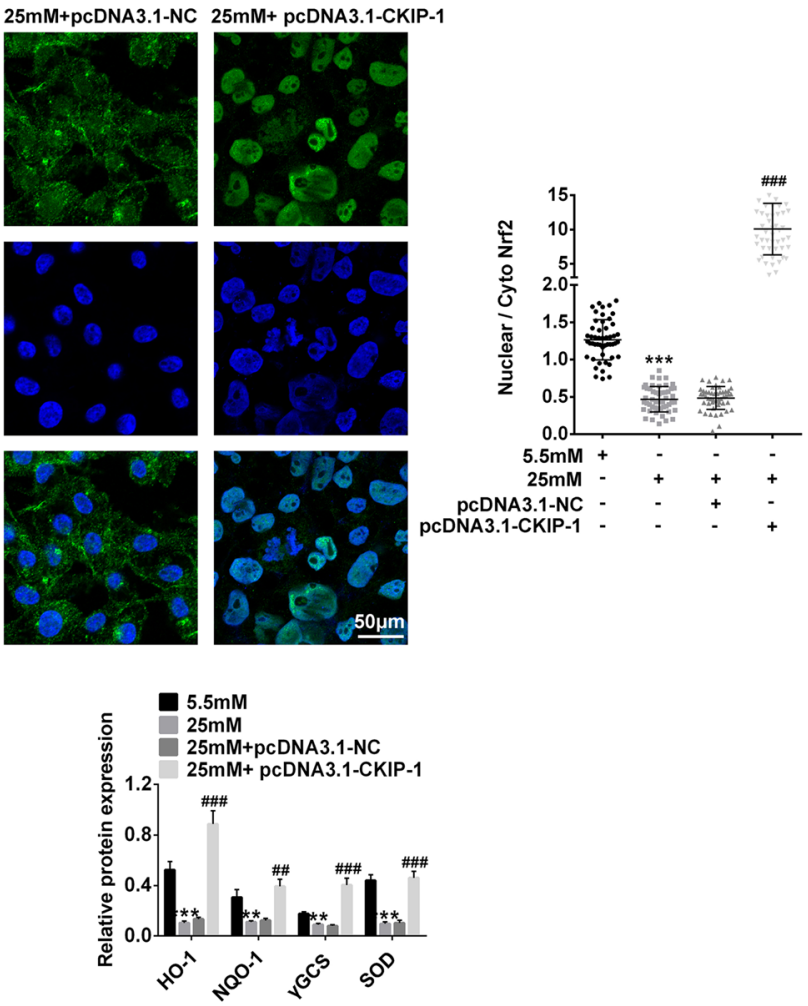

Fig. 4 Nrf2/ARE signaling pathway was activated by CKIP-1 in high-glucose treated HRECs. a, b Co-IP was used to investigate the protein-protein interactions of CKIP-1 and Nrf2 ( $n=3)$. c Luciferase reporter gene system was used to detect the transcriptional activity of Nrf2 $(n=3)$. $\mathbf{d}$ Western Blot was used to detect nuclear Nrf2 and cytoplasm Nrf2 expressions $(n=3)$. e Immunofluorescence assay was performed to detect the expression levels and cellular localization of Nrf2 (Scale bar is $50 \mu \mathrm{m}$ ). f The downstream targets of Nrf2 (including HO-1, NQO-1, YGCS and SOD) were detected by Western Blot $(n=3)$. The data above in one experiments were repeated at least 3 times and performed as mean \pm standard deviation (SD), ${ }^{*} P<0.05$, ${ }^{* *} P<0.01$ and ${ }^{* * *} P<0.001$ 
previous literature [21] and our experiments. Hence we next explored the roles of CKIP-1 and Nrf2/ARE signaling pathway in DR progression. We successfully established Nrf-2 knock-down HRECs models (Fig. 5a, b). Further results showed that overexpressed CKIP-1 abolished the inhibiting effects of high-glucose on cell viability, which were reversed by synergistically knocking down Nrf2 in HRECs (Fig. 5c). In addition to cell viability, CKIP-1 and $N r f 2$ also have similar effects on oxidative stress, inflammation and cell apoptosis. Specifically, the effects of overexpressed CKIP-1 on oxidative stress (MDA levels, SOD activity and GSH-PX activity), inflammation associated cytokines (TNF- $\alpha$, IL- 6 and IL-1 $\beta$ ) secretions and apoptosis in HRECs treated with high-glucose were reversed by synergistically knocking down $N r f 2$ (Fig. 5d-f).

\section{Discussion}

DR is a common complication of DM in clinic, since the underlying mechanisms of DR pathogenesis are still not fully understood, there are still no effective treatments for DR in clinic. Therefore, uncovering the underlying mechanisms might help to cure DR in clinic. DR progression has been reported to be closely related with oxidative stress and inflammation [24], which could be targeted to alleviates DR development in rats [7]. Our clinical experiment results showed that MDA levels was increased, while SOD activity and GSH-PX activity were decreased in DR tissues comparing to the normal tissues, which suggested that oxidative stress was induced in DR tissues. HRECs has been widely used for the investigation of DR progression [25], hence we used high-glucose treated HERCs as the cell models in our experiments to explore the underlying mechanisms of DR pathogenesis in cellular levels. The cellular results were in accordance with the previous study [24] and our clinical results, which showed that high-glucose promotes oxidative stress, cell apoptosis and inflammation, inhibits cell viability. The results above indicated that oxidative stress and inflammation played important roles in DR progression, which might be served as a potential therapeutic agents for DR treatment.

CKIP-1 has been reported to regulate multiple cell functions (cell proliferation [9, 10], apoptosis [11] and differentiation [26]) and involve in the development of several diseases including renal fibrosis [27], chronic heart failure [28], cancer [29] and so on. Recent studies also found that inflammation $[12,13]$ and oxidative stress [11] could be regulated by CKIP-1. For example, CKIP-1 involved in the regulation of immune system and inflammation by regulating microglia M2 polarization [12] and macrophage M1/M2 polarization [13]. Besides, upregulation of CKIP-1 alleviated oxidative stress in cultured hippocampal neurons [11]. However, there are still no literatures reporting the relationship between CKIP-1 and DR pathogenesis. Based on the literatures above, it is reasonable to speculate that CKIP-1 might participate in DR progression by regulating cell viability, oxidative stress and inflammation. The experiment results showed that CKIP-1 is aberrantly downregulated in either DR tissues or high-glucose treated HRECs comparing to the Control group. Further results also showed that overexpressed CKIP-1 reversed the effects of high-glucose treatment on HRECs viability, apoptosis, inflammation associated cytokines secretion and oxidative stress. Similarly, knock-down of CKIP-1 had the opposite effects, which aggravated the effects of high-glucose on HRECs in terms of cell viability, apoptosis, inflammation and oxidative stress. Our cellular results validated our hypothesis and proved that CKIP-1 played a protective role in DR pathogenesis.

Eventhough we have proved that high-glucose induced inhibition of HRECs viability, promotion of HRECs apoptosis, inflammation associated cytokines secretion and oxidative stress, the detailed mechanisms and downstream targets of CKIP-1 are still unknown. Nrf2/ARE signaling is crucial for the regulation of oxidative stress in multiple diseases, such as neurological disorders [30], Parkinson diseases [31] and Alzheimer's disease [32]. Of note, Nrf2/ARE signaling have been reported to be related with DM complications. For example, diabetic nephropathy (DN) could be ameliorated by activating Nrf2/ARE signaling pathway [33] and Nrf2 has been deemed as a potential therapeutic approach to attenuate DR [34]. Our results showed that high-glucose treatment inhibited Nrf2/ARE activation in HRECs. In addition, knock-down of Nrf2 enhanced the inhibiting effects of high-glucose on cell viability and the promoting effects of high-glucose on cell apoptosis, inflammation and oxidative stress. Our results indicated that activation of Nrf2/ARE signaling pathway might help to prevent DR progression, which was in compliance with the previous study [34]. Notably, previous studies have proved that Nrf2/ARE signaling pathway was the downstream target and could be activated by CKIP-1 overexpression [11, 18, $27,35]$. However, it is still unclear whether CKIP-1 alleviates DR development by regulating $N r f 2 / A R E$ signaling pathway. Our results showed that CKIP-1 formed complex with $N r f 2$ and activated Nrf2/ARE signaling pathway. Furthermore, the effects of overexpressed CKIP-1 on high-glucose treated HRECs in terms of cell viability, oxidative stress, inflammation and apoptosis could be abrogated by synergistically knocking down $N r f 2$, which validated our speculation and indicated that CKIP-1 participated in DR development by activating Nrf2/ARE signaling pathway. 

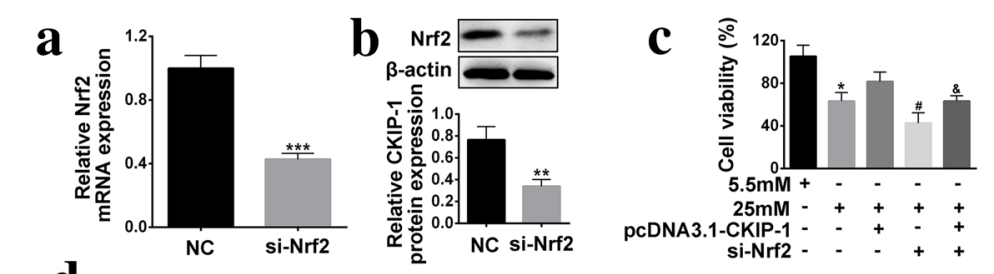

d
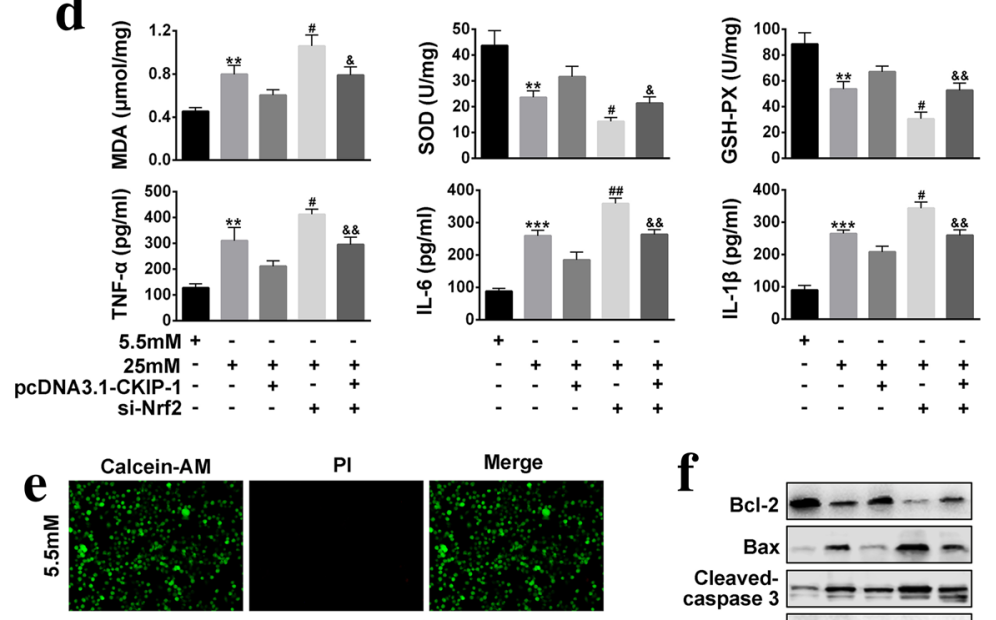

Merge
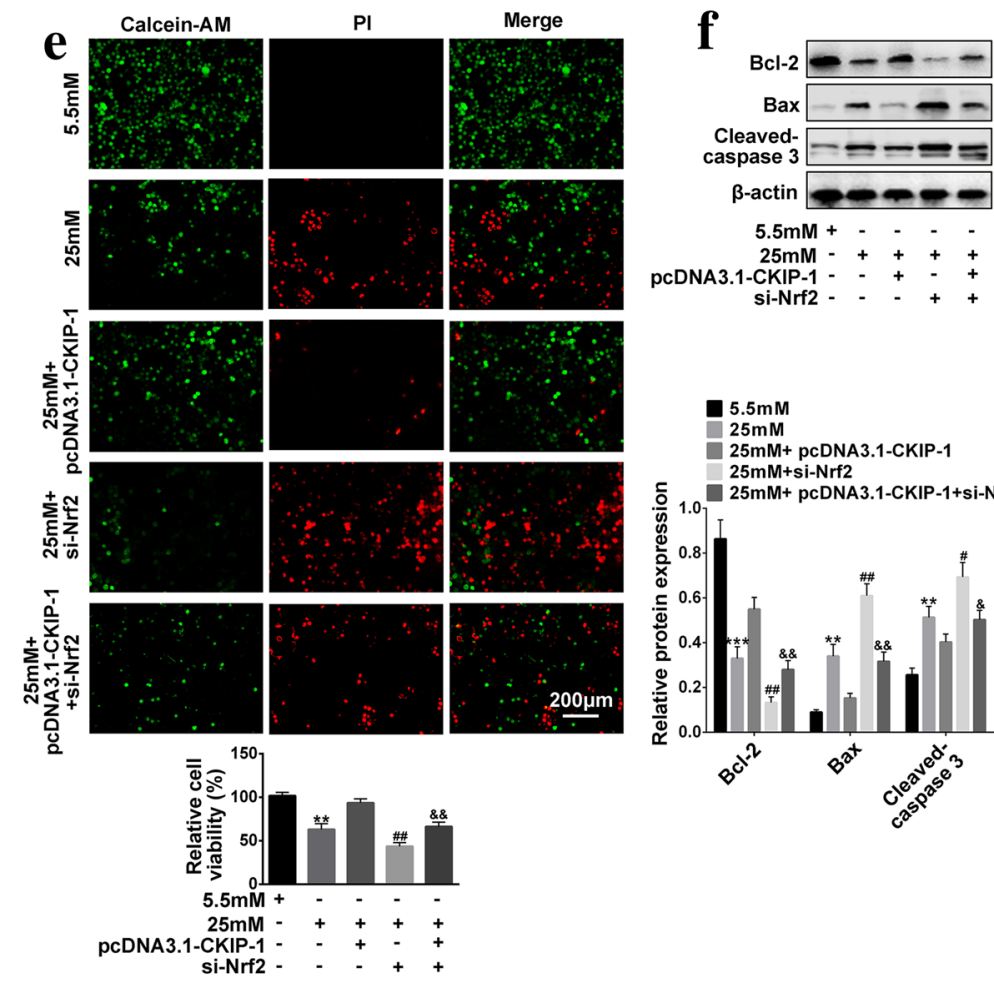

$\begin{array}{ll} & 5.5 \mathrm{mM} \\ & 25 \mathrm{mM} \\ & 25 \mathrm{mM}+\text { pcDNA3.1-CKIP-1 } \\ & 25 \mathrm{mM}+\mathrm{si}-\mathrm{Nrf2} \\ & 25 \mathrm{mM}+\text { pcDNA3.1-CKIP-1+si-Nrf2 }\end{array}$
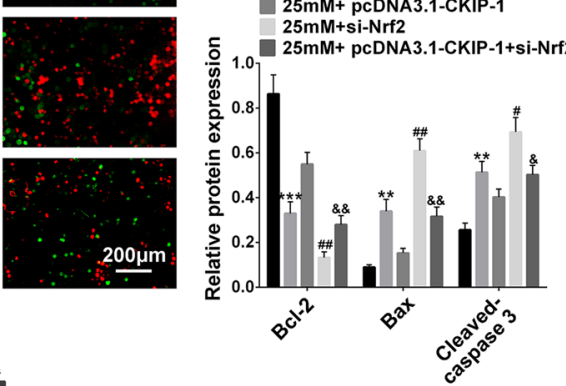

$200 \mu m$

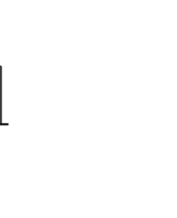

Fig. 5 Knock-down of Nrf2 reverses the effects of overexpressed CKIP-1 on cell viability, oxidative stress, inflammation and apoptosis of high-glucose treated HRECs. a Real-Time qPCR was used to detect relative Nrf2 mRNA levels $(n=3)$. b Western Blot was employed to detect relative Nrf2 protein expressions $(n=3)$. $\mathbf{c}$ Cell viability was evaluated by CCK-8 assay kit $(n=3)$. $\mathbf{d}$ MDA levels, SOD activity and GSH-PX activity were detected by MDA assay kit, SOD kit abd GSH-PX kit respectively $(n=3)$. Inflammation associated cytokines (TNF-a, IL- 6 and IL-1 $\beta$ ) were detected by ELISA $(n=3)$. e Calcein-AM/PI double stain kit was employed to detect cell apoptosis (Scale bar is $200 \mu \mathrm{m})$. $\mathbf{f}$ Apoptosis associated proteins $(B C-2$, Bax and Cleaved Caspase 3) were detected by Western Blot $(n=3)$. The data above in one experiments were repeated at least 3 times and performed as mean \pm standard deviation (SD), ${ }^{*} P<0.05,{ }^{* *} P<0.01$ and ${ }^{* *} P<0.001$

\section{Conclusion}

In conclusion, we proved that overexpressed CKIP-1 could inhibit high-glucose induced HRECs apoptosis, inflammation as well as oxidative stress, and promote
HRECs viability by regulating Nrf2/ARE signaling pathway. Our results will shed light on the discovery of new therapeutic agents for DR treatment in clinic. 


\section{Materials and methods}

\section{Clinical tissue samples}

Human anterior lens capsule tissues were collected from DR patients (Fasting blood glucose $=7.99 \pm 0.53$, $\mathrm{N}=20$, DR group) and normal volunteers (Fasting blood glucose $=5.26 \pm 0.47, \mathrm{~N}=10$, Control group) respectively from Zhejiang Provincial People's Hospital. All the specimens were immediately refrigerated in liquid nitrogen and kept at $-80{ }^{\circ} \mathrm{C}$ for the following experiments. The average age of involved DR patients was $69.23 \pm 7.08$ years old and normal volunteers was $62.80 \pm 7.80$ years old, there was no statistical significance between the two group $(P=0.071)$. The gender (male/ female) in the DR group and Control group were 11/9 and $6 / 4(P=0.794)$ respectively. All the clinical experiments were conducted in accordance with the principle of 'Declaration of Helsinki' [36] and got the approval from the ethics committee of Zhejiang Provincial People's Hospital. Besides, the informed consent had been obtained from all the involved participants. The characteristics of the patients and normal volunteers have been shown on Table 1.

\section{Vectors transfection}

CKIP-1 siRNAs were designed and synthesized by Shanghai GenePharma Co., Ltd (Shanghai, China), the sequences of the siRNAs were showed as follows, siCKIP-1 \#1: 5'-CCGCUAUGUGGUGCUGAAA-3'; si-CKIP-1 \#2: 5'-GGAACCAACCUCUUGUGCU-3'. Besides, the cDNA fragments of CKIP-1 and pcDNA3.1 vectors were digested and prepared by EcoRV/XhoI double enzymes digestion. After that, the CKIP-1 cDNA fragments were inserted into pcDNA3.1 vectors by T4 DNA ligase (Thermo Fisher Scientific, Waltham, MA, USA) to generate pcDNA3.1/CKIP-1 overexpression vectors. Besides, siRNAs of $N r f 2$ was constructed according to the previous study [37], the siRNA sequence of $N r f 2$ was 5'-UAAUUGUCAACUACUGUCAGUU-3'. After the cell confluence reached approximate $80 \%$, all the overexpressed vectors of siRNAs were transfected into HRECs by using Lipofectamine RNAiMAX Reagent (Invitrogen, Carlsbad, CA, USA) under the instructions of the manufacturer. After transfection, Real-Time qPCR and Western Blot were used to detect the efficiency of the above overexpressed vectors or siRNAs. After $24 \mathrm{~h}$ transfection, the culture medium was replaced with the medium containing high-glucose $(25 \mathrm{mM})$ and co-cultured for $48 \mathrm{~h}$.

\section{Cell culture and Cell counting kit-8 (CCK-8) assay}

Human retinal endothelial cells (HRECs) were purchased from Angioproteomie company (Boston, MA, USA), which has been used in the previous study [38].

\begin{tabular}{llll}
$\begin{array}{l}\text { Table } 1 \text { Characteristics of the patient and the control } \\
\text { group }\end{array}$ & Control $(\mathbf{n}=\mathbf{1 0})$ & $\begin{array}{l}\text { Diabetic } \\
\text { cataract } \\
(\mathbf{n}=\mathbf{2 0})\end{array}$ & P value \\
\hline & & $70.5 \pm 5.31$ & 0.068 \\
\hline Age (years) & $66.70 \pm 4.85$ & $12 / 8$ & 0.592 \\
$\begin{array}{l}\text { Gender (male/female) } \\
\begin{array}{l}\text { Fasting blood glucose } \\
\text { (mmol/l) }\end{array}\end{array}$ & $7 / 3$ & $8.01 \pm 0.67$ & $<0.001$ \\
\hline
\end{tabular}

According to the company's instruction, HRECs were cultured in endothelial cell medium (Sciencell, Carlsbad, CA, USA) supplemented with $5 \%$ fetal bovine serum (FBS), $100 \mu \mathrm{g} / \mathrm{ml}$ streptomycin (Wuhan Fortuna Chemical Co., Ltd, Wuhan, China). After transfecting cells with different vectors, cells were diluted to $1 \times 10^{4}$ per well in the six-well plates and cultured in the incubator (Thermo Fisher Scientific, Waltham, MA, USA) under the temperature of $37{ }^{\circ} \mathrm{C}$ and a humidified atmosphere containing $5 \% \mathrm{CO}_{2}$ and air. The cells were then exposed to $5.5 \mathrm{mM}$ and $25 \mathrm{mM}$ glucose for $48 \mathrm{~h}$. Cell morphology was observed by inverted microscope (Olympus Corporation, Japan).

Cell viability was evaluated by CCK- 8 assay according to the manufacturer's instruction. In brief, HRECs were diluted and seeded in the 96-well plate in the concentration of 5000 cells per well. The CCK- 8 solution $(10 \mu \mathrm{l}$ per well) was then added into the wells and incubated with the cells for $2 \mathrm{~h}$ in the standard culture conditions. Finally, the optical density (OD) values were detected and quantified in the wavelength of $450 \mathrm{~nm}$ to evaluate cell viability.

\section{Real-Time qPCR}

The total RNA was extracted from DR tissues or highglucose treated HRECs by Trizol reagent (Invitrogen corporation, Carlsbad, California, USA) according to the manufacturer's instruction. The extracted total RNA was then reversely transcribed to complementary DNA (cDNA) by using TaqMan Reverse Transcription Reagents (Applied Biosystems, Foster City, CA, USA). The SYBR Green PCR Master Mix (Applied Biosystems, Thermo Fisher Scientific, Waltham, MA, USA) was employed to perform Real-Time qPCR to quantify the target cDNA by using the Applied Biosystems 7500 RealTime qPCR System. The procedures were set as $95^{\circ} \mathrm{C}$ for $10 \mathrm{~min}$ and 40 cycles of $95^{\circ} \mathrm{C}$ for $15 \mathrm{~s}$ and $60^{\circ} \mathrm{C}$ for $1 \mathrm{~min}$ according to the previous study. The primer sequences of the related genes are listed in the following Table 2. The relative mRNA expression levels were normalized to $\beta$-actin by using the $2^{-\Delta \Delta C t}$ method. 


\section{Western Blot}

The total proteins in the clinical tissues and HRECs were extracted by using RIPA lysis buffer (Beyotime Biotechnology, Shanghai, China) according to the manufacturer's instruction. The nuclear fractions were extracted by using the Nuclear/Cytosol Fractionation Kit (BioVision, Inc., Milpitas, CA, USA) according to the previous study [39]. The lysates were obtained by centrifugation and protein concentrations were quantified by using a BCA kit (Beyotime Biotechnology, Shanghai, China). The $10 \%$ SDS-polyacrylamide gel was used to separate the target proteins, which were then transferred to PVDF membranes (Millipore, Bedford, MA, USA). After blocking the membranes with 5\% skim milk diluted in TBST (Tris-buffered saline containing 0.1\% Tween-20) for $45 \mathrm{~min}$ at $37^{\circ} \mathrm{C}$, the membranes were then incubated with the primary antibodies against $\beta$-actin (1:2000, \#ab8226, Abcam, UK), CKIP-1 (1:1000, \#ab91489, Abcam, UK), Nrf2 (1:1000, \#ab137550, Abcam, UK), Bcl-2 (1:1000, \#ab32124, Abcam, UK), cleaved Caspase 3 (1:1000, \#ab13847, Abcam, UK), Bax (1:1000, \#ab53154, Abcam, UK), Lamin B (1:500, \#ab133741, Abcam, UK), Tublin (1:2000, \#ab18251, Abcam, UK), HO-1 (1:1000, \#ab13248, Abcam, UK), NQO-1 (1:1000, \#ab80588, Abcam, UK), $\gamma$ GCS (1:1000, \#ab59956, Abcam, UK) and SOD (1:1000, \#ab80946, Abcam, UK) at $4{ }^{\circ} \mathrm{C}$ overnight. The membranes were next washed with TBST three times and incubated with horseradish peroxidase (HRP)conjugated secondary antibody (Abcam) for $1 \mathrm{~h}$ at room temperature. After that, ECL Western Blotting Detection Kit (GE Healthcare Bio-Science, Pittsburgh, PA, USA) was next purchased to detect the protein bands, the optical density of the protein bands were quantified by Image J software and normalized to $\beta$-actin.

\section{Detection of oxidative stress by MDA assay kit, SOD kit and GSH-Px assay kit}

Malondialdehyde (MDA) was the degraded product of polyunsaturated lipids, which could be used to measure the level of lipid peroxidation and oxidative stress. MDA could react with thiobarbituric acid as a thiobarbituric acid reactive substances (TBARS) to form a 1:2 MDATBA adduct, hence measuring the contents of TBARS

Table 2 The primers of the involved genes

\begin{tabular}{ll}
\hline Gene & Primer sequences (strand) \\
\hline$\beta$-Actin & Forward: 5'-CTCCATCCTGGCCTCGCTGT-3' \\
& Reverse: 5'-GCTGCTACCTTCACCGTTCC-3' \\
CKIP-1 & Forward: 5'-AATTCTGCGGGAAAGGGATTT-3' \\
& Reverse: 5'-AACACCTCCTGACTGTTTTTCTC-3' \\
Nrf2 & Forward: 5'-GACCTAAAGCACAGCCAACACAT-3' \\
& Reverse: 5'-CTCAATCGGCTTGAATGTTTGTC-3'
\end{tabular}

reflected MDA levels. The MDA assay kit (Beyotime Biotechnology, Shanghai, China) was used to evaluate TBARS according to the manufacturer's instruction.

Superoxide dismutase (SOD) played an important role in the regulation of oxidative stress, which could help to eliminate reactive oxygen species (ROS) and protect cells from the damage of oxidative stress. Hence, SOD activity could be used as a biomarker to evaluate oxidative stress in cells. The SOD assay kit (Nanjing Jiancheng Bioengineering Institute, Nanjing, China) was used to detect SOD activity in high-glucose treated HRECs according to the manufacturer's instruction.

Glutathione peroxidase (GSH-PX) was an important antioxidant enzyme in cells, which could be used to evaluate the levels of oxidative stress. The GSH-PX assay kit (Nanjing Jiancheng Bioengineering Institute, Nanjing, China) was used to estimate GSH-PX activity in highglucose treated HRECs according to the manufacturer's instruction. The absorbance of the samples was determined at $412 \mathrm{~nm}$ for GSH-PX at the end of reaction on a microplate reader.

\section{Detection of cell apoptosis by Calcein-AM/PI double stain kit}

Calcein-AM/propidium iodide (PI) double stain kit (Shanghai Yeasen Corporation, Shanghai, China) was used to detect cell apoptosis according to the manufacturer's instruction. In brief, high-glucose treated HRECs were washed with assay buffer for 3 times, and cells were suspended with assay buffer at the density of $1 \times 10^{5} / \mathrm{ml}$. After that, the working reagent containing Calcein-AM and PI at the ratio of 4:3 was added and incubated with the cells in $37{ }^{\circ} \mathrm{C}$ for $15 \mathrm{~min}$. The inverted fluorescence microscope (Olympus Corporation, Japan) was employed to observe HRECs apoptosis ratio.

\section{Enzyme-linked ImmunoSorbent Assay (ELISA)}

The inflammation associated cytokines (TNF- $\alpha$, IL- 6 and IL-1 $\beta$ ) in the experiments were detected by TNF- $\alpha$ ELISA Kit (\#ab181421, Abcam, UK),IL-6 ELISA Kit (\#ab46027, Abcam, UK) and IL-1ß ELISA Kit (\#ab46052, Abcam, UK) respectively. In brief, the culture supernants in the Control group and high-glucose treated group were collected. The expression levels of TNF- $\alpha$, IL- 6 and IL-1 $\beta$ were detected according to the manufacturer's instruction.

\section{Co-immunoprecipitation (CO-IP)}

The Flag-labeled CKIP-1 (Flag-CKIP-1) and HA-labeled Nrf2 (HA-Nrf2) plasmids were used in the CO-IP 
experiment. The Flag-CKIP-1 plasmid or HA-Nrf2 plasmid was transfected into the HRECs by using the Lipofectamine RNAiMAX Reagent (Invitrogen, Carlsbad, CA, USA) according to the manufacturer's protocol. After vectors transfection for $24 \mathrm{~h}$, the total proteins in HRECs were extracted by using RIPA lysis buffer (Beyotime Biotechnology, Shanghai, China) according to the manufacturer's instruction. The supernatant was collected and incubated with protein A-Sepharose (Sigma, USA) at $4{ }^{\circ} \mathrm{C}$ for $1 \mathrm{~h}$. The following sodium dodecyl sulfate polyacrylamide gel electrophoresis (SDS-PAGE) was next used to separate the target proteins. After that, the anti-Flag (1:1000, Sigma, USA) and anti-HA (1:1000, Sigma, USA) were used as the primary antibodies for further immunodetection. Besides, the peroxidase-conjugated anti-rabbit immunoglobulin G (IgG) (1:2000, \#ab133470, Abcam, UK) was used as the secondary antibody. ECL Western Blotting Detection Kit (GE Healthcare Bio-Science, Pittsburgh, PA, USA) was next used to quantify the protein contents.

\section{Luciferase reporter gene system}

The ARE promoter fragment was subcloned into pGL3 basic vector to produce pGL3-ARE luciferase vectors, which were then transfected into HRECs by using the Lipofectamine RNAiMAX Reagent (Invitrogen, Carlsbad, CA, USA). The HRECs were then treated with low-glucose $(5.5 \mathrm{mM})$, high-glucose $(25 \mathrm{mM})$, high-glucose and pcDNA3.1-NC vectors, high-glucose and pcDNA3.1CKIP-1 vectors respectively. The $\mathrm{pRL}-\mathrm{TK}$ vector was next used to normalized the transfection efficiency. After that, the HRECs were lysed by RIPA lysis buffer (Beyotime Biotechnology, Shanghai, China) for further analysis. The dual-luciferase reporter assay system (Promega, Madison, Wis., USA) was used to measure luciferase activity according to the manufacturer's protocol.

\section{Statistical analysis}

All the experiments were conducted at least for three times. The data was collected and showed as mean \pm standard deviation (SD) for further analysis. Student's t test was used to compare the two groups in the experiments. One way analysis of variance (ANOVA) was used to compare multiple groups (more than 3 groups) in the experiments. Statistical analysis was performed using the SPSS 18.0 software. Differences of $\mathrm{P}<0.05$ were considered as statistical significance.

\section{Supplementary information}

Supplementary information accompanies this paper at https://doi. org/10.1186/s13578-019-0331-x.
Additional file 1: Figure S1. The effects of high-glucose treatment on cell viability and inflammation. $(A, C)$ Cell viability was detected by using CCK-8 assay. (B) The inflammatory cytokines (TNF- $\alpha$, IL- 6 and IL-1 $\beta$ ) were detected by using an ELISA kit. The data above in one experiments were repeated at least 3 times and performed as mean \pm standard deviation (SD), ${ }^{*} P<0.05,{ }^{*} P<0.01$ and ${ }^{* *} P<0.001$.

Additional file 1: Figure $\mathbf{S 2}$. The schematic model of this study.

\section{Abbreviations}

MDA: malondialdehyde; SOD: superoxide dismutase; GSH-Px: glutathione peroxidase; ELISA: Enzyme-linked ImmunoSorbent Assay; CO-IP: co-immunoprecipitation; DR: diabetic retinopathy; DM: diabetes mellitus; CKIP-1: casein kinase 2 interacting protein-1; Nrf2: nuclear factor E2-related factor 2; ARE: antioxidant response element; /CAM-1: intercellular cell adhesion molecule-1.

\section{Acknowledgements}

Not applicable.

\section{Authors' contributions}

$\mathrm{LZ}$ and $\mathrm{HZ}$ conceived and designed the experiments, JY analyzed and interpreted the results of the experiments, MY performed the experiments. All authors read and approved the final manuscript.

\section{Funding}

Funding information is not applicable.

Availability of data and materials

All data generated or analyzed during this study are included in this published article (Additional file 2).

Ethics approval and consent to participate

Not applicable.

\section{Consent for publication}

Not applicable.

\section{Competing interests}

The authors declare that they have no competing interests.

\section{Author details \\ ${ }^{1}$ Department of Ophthalmology, Zhejiang Provincial People's Hospital, No.158, Shangtang Road, Xiacheng District, Hangzhou 310014, Zhejiang, China. ${ }^{2}$ Department of Ophthalmology, People's Hospital of Hangzhou Medi- cal College, No.128, ShangTang Road, XiaCheng District, Hangzhou 310014, Zhejiang, China.}

Received: 29 May 2019 Accepted: 15 August 2019

Published online: 23 August 2019

\section{References}

1. Jenkins AJ, Joglekar MV, Hardikar AA, Keech AC, O'Neal DN, Januszewski AS. Biomarkers in diabetic retinopathy. Rev Diabetic Stud. 2015;12(1-2):159-95.

2. Ung C, Sanchez AV, Shen L, Davoudi S, Ahmadi T, Navarro-Gomez $D$, et al. Whole exome sequencing identification of novel candidate genes in patients with proliferative diabetic retinopathy. Vision Res. 2017;139:168-76

3. Zhang ZZ, Qin XH, Zhang J. MicroRNA-183 inhibition exerts suppressive effects on diabetic retinopathy by inactivating BTG1-mediated PI3K/Akt/VEGF signaling pathway. Am J Physiol Endocrinol Metab. 2019;316:E1050-60.

4. Shao Y, Dong LJ, Takahashi Y, Chen J, Liu X, Chen Q, et al. miRNA-451a regulates RPE function through promoting mitochondrial function in proliferative diabetic retinopathy. Am J Physiol Endocrinol Metab. 2019;316(3):E443-52 
5. Calderon GD, Juarez OH, Hernandez GE, Punzo SM, De la Cruz ZD. Oxidative stress and diabetic retinopathy: development and treatment. Eye (London, England). 2017;31(8):1122-30.

6. Rubsam A, Parikh S, Fort PE. Role of inflammation in diabetic retinopathy. Int J Mol Sci. 2018;19(4):E942.

7. Ola MS, Alhomida AS, LaNoue KF. Gabapentin attenuates oxidative stress and apoptosis in the diabetic rat retina. Neurotox Res. 2019;36:81-90.

8. Ren C, Wu H, Li D, Yang Y, Gao Y, Jizhang Y, et al. Remote ischemic conditioning protects diabetic retinopathy in streptozotocin-induced diabetic rats via anti-inflammation and antioxidation. Aging Dis. 2018;9(6):1122-33.

9. Zhang L, Wang $Y$, Xiao F, Wang $S$, Xing G, Li Y, et al. CKIP-1 regulates macrophage proliferation by inhibiting TRAF6-mediated Akt activation. Cell Res. 2014;24(6):742-61.

10. Zhu X, Ouyang Y, Zhong F, Wang Q, Ding L, Zhang P, et al. Silencing of CKIP-1 promotes tumor proliferation and cell adhesion-mediated drug resistance via regulating AKT activity in non-Hodgkin's lymphoma. Oncol Rep. 2017;37(1):622-30.

11. Xiang Y, Fan X, Zhao M, Guo Q, Guo S. CKIP-1 alleviates oxygen-glucose deprivation/reoxygenation-induced apoptosis and oxidative stress in cultured hippocampal neurons by downregulating Keap1 and activating Nrf2/ARE signaling. Eur J Pharmacol. 2019;848:140-9.

12. Yang $Z$, Jiang $X$, Zhang J, Huang $X$, Zhang $X$, Wang J, et al. Let-7a promotes microglia M2 polarization by targeting CKIP-1 following ICH. Immunol Lett. 2018;202:1-7.

13. Chen Y, Liu W, Wang Y, Zhang L, Wei J, Zhang X, et al. Casein Kinase 2 Interacting Protein-1 regulates $M 1$ and $M 2$ inflammatory macrophage polarization. Cell Signal. 2017;33:107-21.

14. Liao W, Fu Z, Zou Y, Wen D, Ma H, Zhou F, et al. MicroRNA-140-5p attenuated oxidative stress in Cisplatin induced acute kidney injury by activating Nrf2/ARE pathway through a Keap1-independent mechanism. Exp Cell Res. 2017;360(2):292-302.

15. Haithloul HAS, Alotaibi MF, Bin-Jumah M, Elgebaly H, Mahmoud AM. Olea europaea leaf extract up-regulates Nrf2/ARE/HO-1 signaling and attenuates cyclophosphamide-induced oxidative stress, inflammation and apoptosis in rat kidney. Biomed Pharmacother. 2019;111:676-85.

16. Chang J, Zhang Y, Li Y, Lu K, Shen Y, Guo Y, et al. NrF2/ARE and NF-kappaB pathway regulation may be the mechanism for lutein inhibition of human breast cancer cell. Fut Oncol (London, England). 2018;14(8):719-26.

17. Jin XF, Wang $S$, Shen M, Wen X, Han XR, Wu JC, et al. Effects of rehabilitation training on apoptosis of nerve cells and the recovery of neural and motor functions in rats with ischemic stroke through the PI3K/Akt and Nrf2/ARE signaling pathways. Brain Res Bull. 2017;134:236-45.

18. Gong W, Chen C, Xiong F, Yang Z, Wang Y, Huang J, et al. CKIP-1 ameliorates high glucose-induced expression of fibronectin and intercellular cell adhesion molecule-1 by activating the Nrf2/ARE pathway in glomerular mesangial cells. Biochem Pharmacol. 2016;116:140-52.

19. Li S, Yang H, Chen X. Protective effects of sulforaphane on diabetic retinopathy: activation of the Nrf2 pathway and inhibition of NLRP3 inflammasome formation. Exp Anim. 2019;68:221-31.

20. Bucolo C, Drago F, Maisto R, Romano GL, D'Agata V, Maugeri G, et al. Curcumin prevents high glucose damage in retinal pigment epithelial cells through ERK1/2-mediated activation of the Nrf2/HO-1 pathway. J Cell Physiol. 2019;23:17295-304.

21. Long M, Yang SH, Shi W, Li P, Guo Y, Guo J, et al. Protective effect of proanthocyanidin on mice Sertoli cell apoptosis induced by zearalenone via the Nrf2/ARE signalling pathway. Environ Sci Pollut Res Int. 2017;24(34):26724-33.

22. Gao Y, Chu SF, Zhang Z, Ai QD, Xia CY, Huang HY, et al. Ginsenoside Rg1 prevents acetaminophen-induced oxidative stress and apoptosis via Nrf2/ARE signaling pathway. J Asian Nat Prod Res. 2019;21:782-97.

23. Velagapudi R, Kumar A, Bhatia HS, El-Bakoush A, Lepiarz I, Fiebich BL, et al. Inhibition of neuroinflammation by thymoquinone requires activation of Nrf2/ARE signalling. Int Immunopharmacol. 2017:48:17-29.

24. Dehdashtian E, Mehrzadi S, Yousefi B, Hosseinzadeh A, Reiter RJ, Safa M, et al. Diabetic retinopathy pathogenesis and the ameliorating effects of melatonin; involvement of autophagy, inflammation and oxidative stress. Life Sci. 2018;193:20-33.
25. Thomas AA, Feng B, Chakrabarti S. ANRIL: a regulator of VEGF in diabetic retinopathy. Invest Ophthalmol Vis Sci. 2017;58(1):470-80.

26. Song Y, Wang C, Gu Z, Cao P, Huang D, Feng G, et al. CKIP-1 suppresses odontoblastic differentiation of dental pulp stem cells via BMP2 pathway and can interact with NRP1. Connect Tissue Res. 2019;60(2):155-64.

27. Gong W, Chen Z, Zou Y, Zhang L, Huang J, Liu P, et al. CKIP-1 affects the polyubiquitination of Nrf2 and Keap1 via mediating Smurf1 to resist HG-induced renal fibrosis in GMCs and diabetic mice kidneys. Free Radical Biol Med. 2018;115:338-50.

28. Li MP, Zhang YJ, Hu XL, Zhou JP, Yang YL, Peng LM, et al. Association of CKIP-1 P21A polymorphism with risk of chronic heart failure in a Chinese population. Oncotarget. 2017;8(22):36545-52.

29. Nie J, Liu L, Xing G, Zhang M, Wei R, Guo M, et al. CKIP-1 acts as a colonic tumor suppressor by repressing oncogenic Smurf1 synthesis and promoting Smurf1 autodegradation. Oncogene. 2014;33(28):3677-87.

30. Vasconcelos AR, Dos Santos NB, Scavone C, Munhoz CD. Nrf2/ARE pathway modulation by dietary energy regulation in neurological disorders. Front Pharmacol. 2019;10:33.

31. Gureev AP, Popov VN. Nrf2/ARE pathway as a therapeutic target for the treatment of parkinson diseases. Neurochem Res. 2019. https://doi. org/10.1007/s11064-018-02711-2.

32. Tian $Y$, Wang W, Xu L, Li H, Wei Y, Wu Q, et al. Activation of Nrf2/ARE pathway alleviates the cognitive deficits in PS1V97L-Tg mouse model of Alzheimer's disease through modulation of oxidative stress. J Neurosci Res. 2019;97(4):492-505.

33. Chen YJ, Kong L, Tang ZZ, Zhang YM, Liu Y, Wang TY, et al. Hesperetin ameliorates diabetic nephropathy in rats by activating Nrf2/ARE/glyoxalase 1 pathway. Biomed Pharmacother. 2019;111:1166-75.

34. Deliyanti D, Alrashdi SF, Tan SM, Meyer C, Ward KW, de Haan JB, et al. Nrf2 activation is a potential therapeutic approach to attenuate diabetic retinopathy. Invest Ophthalmol Vis Sci. 2018;59(2):815-25.

35. Gong W, Li J, Chen Z, Huang J, Chen Q, Cai W, et al. Polydatin promotes Nrf2-ARE anti-oxidative pathway through activating CKIP-1 to resist HG-induced up-regulation of FN and ICAM-1 in GMCs and diabetic mice kidneys. Free Radical Biol Med. 2017;106:393-405.

36. Issue Information-Declaration of Helsinki. J Bone Min Res 2017;32(3):BMi-BMii.

37. Li PC, Tu MJ, Ho PY, Jilek JL, Duan Z, Zhang QY, et al. Bioengineered NRF2-siRNA is effective to interfere with NRF2 pathways and improve chemosensitivity of human cancer cells. Drug Metab Disposit. 2018;46(1):2-10.

38. Zhang Y, LV X, Hu Z, Ye X, Zheng X, Ding Y, et al. Protection of Mcc950 against high-glucose-induced human retinal endothelial cell dysfunction. Cell Death Dis. 2017;8(7):e2941.

39. Fang Y, Zhao Y, He S, Guo T, Song Q, Guo N, et al. Overexpression of FGF19 alleviates hypoxia/reoxygenation-induced injury of cardiomyocytes by regulating GSK-3beta/Nrf2/ARE signaling. Biochem Biophys Res Commun. 2018;503(4):2355-62.

\section{Publisher's Note}

Springer Nature remains neutral with regard to jurisdictional claims in published maps and institutional affiliations.

Ready to submit your research? Choose BMC and benefit from:

- fast, convenient online submission

- thorough peer review by experienced researchers in your field

- rapid publication on acceptance

- support for research data, including large and complex data types

- gold Open Access which fosters wider collaboration and increased citations

- maximum visibility for your research: over $100 \mathrm{M}$ website views per year

At BMC, research is always in progress.

Learn more biomedcentral.com/submissions 